\title{
In situ Microscopy Studies of Liquid Gallium Droplet Dynamics
}

\author{
Chilan Ngo and S. Kodambaka
}

Department of Materials Science and Engineering, University of California Los Angeles, Los Angeles, CA, 90095.

Liquid metals such as gallium and related alloys are promising as hydrogen- and energy-storage materials [1], for battery electrodes [2], in mercury-free thermometry [3], as plasmonic coupling materials [4], in electrical switches [5], as conductors in ultra-stretchable wires [6], and as catalysts for the growth of nanowires [7-8], and graphene [9-10]. This is largely due to their low vapor pressure, high wettability, and good thermal and electrical conductivities. All of these applications rely on understanding the thermochemical stability of liquid-metal/solid interfaces, since it affects adhesion, wettability, chemical reactivity, and optoelectronic properties. Here, we present results from in situ variable-temperature transmission electron microscopy (TEM) investigations of the thermal stability of liquid gallium on solid surfaces. From time- and temperature-dependent measurements of the observed droplet size changes, we can describe the mass transport mechanisms controlling the Ga droplet dynamics.

Our in situ TEM experiments are carried out using cross-sectional TEM (XTEM) and plan-view TEM samples. XTEM samples implanted with $\mathrm{Ga}^{+}$are prepared by focused ion beam (FIB) milling of $\mathrm{Al}_{2} \mathrm{O}_{3}(0001)$ substrates via FEI Nova Nano 600 Dual-Beam system. Plan-view samples are prepared by depositing pure Ga via GEN 930 molecular beam epitaxy (MBE) system onto TEM grids with amorphous $\mathrm{SiO}_{2}$ or $\mathrm{C}$ support films. Both types of samples are annealed in vacuum $\left(8 \times 10^{-8}\right.$ Torr $)$ at temperatures between 300 and $700{ }^{\circ} \mathrm{C}$, using a Hummingbird heating holder in FEI Technai 12 and Titan S/TEM systems for times up to $13 \mathrm{~h}$.

We find that annealing the XTEM samples at moderate temperatures $\left(\mathrm{T} \geq 200{ }^{\circ} \mathrm{C}\right)$ results in the formation of spherical droplets of Ga on amorphous $\mathrm{C}$ films, which undergo coarsening/decay (Ostwald ripening) at temperatures up to $500{ }^{\circ} \mathrm{C}$ [11]. In addition to Ostwald ripening, we observe a new phenomenon: "regeneration" of Ga droplets during annealing. In this process, a smaller Ga droplet nucleates and grows at one site, is consumed by a larger adjacent droplet, and is replaced by a similar droplet growing at the same location. Fig. 1 shows regeneration of one such droplet during annealing of an XTEM sample at $\mathrm{T}=400{ }^{\circ} \mathrm{C}$. In this image sequence, the droplet is approximately half of its maximum size at $\Delta t=0$. Its size decreases with increasing time, disappears at $\Delta t=16 \mathrm{~s}$, and is shown growing again at $\Delta t=18 \mathrm{~s}$. We find that this phenomenon produces multiple cycles of droplet regrowth. Although disappearance of the small droplet can be attributed to coalescence with the larger one, the periodic regeneration of another droplet at the same site is unusual. Previous studies indicate that FIB milling leads to $\mathrm{Ga}^{+}$implantation and creation of surface defects that act as preferential sites for nucleation [12]. Thus, it is likely that Ga implanted within the substrate diffuses out of these sites and forms $\mathrm{Ga}$ droplets. We envision that the out-flux of $\mathrm{Ga}$ is high and constant, giving rise to the observed periodic regeneration of droplets.

We also find that annealing MBE-deposited Ga droplets at $\mathrm{T}>600{ }^{\circ} \mathrm{C}$ causes evaporation. Although ultrapure Ga is deposited in a UHV system, the samples are stored under ambient conditions before being air-transferred into the TEM, which likely causes the formation of an oxide. During evaporation 
of these droplets, we find that the decay rates vary with time and with droplet size. For example, a few of the larger droplets $(\mathrm{r}>450 \mathrm{~nm})$ decrease in size monotonically from $t=0$, whereas smaller droplets $(\mathrm{r}$ $<450 \mathrm{~nm}$ ) do not change significantly in size until $t>200 \mathrm{~min}$, after which there is a distinct increase in their rates of evaporation. We speculate that the observed time- and size-dependent variations are due to the presence of oxide shells around the droplets, which is an ongoing point of study. In summary, our in situ TEM studies of gallium droplets help develop insights into the thermochemical stability and behavior of liquid metals on solid surfaces [13].

\section{References:}

[1] B Sakintuna et al, Int. J. Hydrogen Energy, 32 (2007), p. 1121; DJ Bradwell et al, J. Am. Chem. Soc., 134 (2012), p. 1895.

[2] D Deshpande et al, J. Electrochem. Soc. 158 (2011), p. A845.

[3] Y Gao and Y Bando, Nature 415 (2002), p. 599; YB Li et al, App. Phys. Lett. 83 (2003), p. 999.

[4] SRC Vivekchand et al, Nano Lett. 12 (2012), 4324-4328; CW Yi et al, Small 8 (2012), p. 2721.

[5] PS Dorozhkin et al, Small 1 (2005), p. 1088.

[6] S Zhu et al, Adv. Funct. Mater. 23 (2013), p. 2308.

[7] ZW Pan et al, J. Am. Chem. Soc. 124 (2002), p. 1817.

[8] KA Dick, Prog. Cryst. Growth Charact. Mater. 54 (2008), p. 138.

[9] J Fujita et al, J. Vac. Sci. Technol. B 27 (2009), p. 3063.

[10] H Hiura et al, Carbon 50 (2012), p. 5076.

[11] S Kodambaka et al, App. Phys. Lett. 102 (2013), p. 161601.

[12] P Phillipp et al, Nanotech. 23 (2012), p. 475304.

[13] We gratefully acknowledge support from the AFOSR (Dr. Ali Sayir) FA9550-10-1-0496 and from the NSF through the Grant No. CMMI-1200547. We thank Mr. Noah Bodzin and the Nanoelectronics Research Facility in the UCLA HSSEAS for assistance with FIB milling, Dr. Baolai Liang and the Integrated NanoMaterials Lab at CNSI for MBE deposition, and Dr. Justinas Palisaitis for conducting energy dispersive spectroscopy at Linköping University. The authors acknowledge the use of instruments at the Electron Imaging Center for NanoMachines supported by NIH (1S10RR23057 to ZHZ) and CNSI at UCLA.
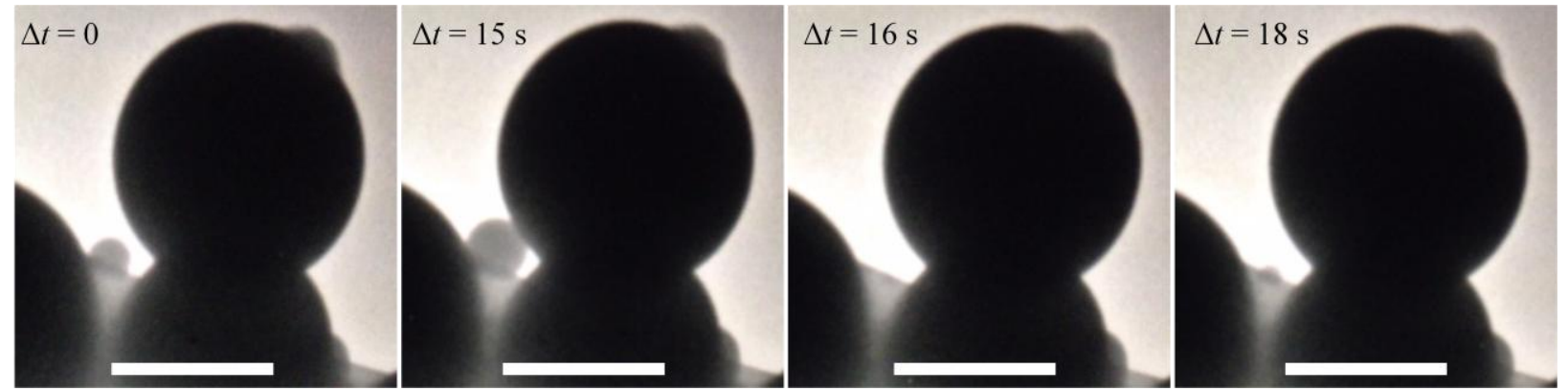

Figure 1. Typical bright-field TEM images of Ga droplets acquired at $\mathrm{T}=400{ }^{\circ} \mathrm{C}$. The small droplet to the left grows on top of the substrate at times between $\Delta t=0$ and $15 \mathrm{~s}$, disappears at $\Delta t=16 \mathrm{~s}$, and has begun growing again at $\Delta t=18 \mathrm{~s}$. Scale bars $=500 \mathrm{~nm}$. 